\title{
A Very Low Level dc Current Amplifier Using SC Circuit: Effects of Parasitic Capacitances and Duty Ratio on Its Output
}

\author{
Hiroki Higa, Ryota Onaga, Naoki Nakamura \\ Faculty of Engineering, University of the Ryukyus, Okinawa, Japan \\ Email: hrhiga@eve.u-ryukyu.ac.jp
}

Received 5 June 2014; revised 22 July 2014; accepted 3 August 2014

Copyright (C) 2014 by authors and Scientific Research Publishing Inc.

This work is licensed under the Creative Commons Attribution International License (CC BY). http://creativecommons.org/licenses/by/4.0/

cc) (7) Open Access

\section{Abstract}

This paper describes a very low level dc current amplifier using switched capacitor (SC) circuit to miniaturize and improve its output response speed, instead of the conventionally used high-ohmage resistor. A switched capacitor filter (SCF) and an offset controller are also used to decrease vibrations and offset voltage at the output of the amplifier. The simulation results show that the parasitic capacitances that are distributed to the input portion of the amplifier have some effect on offset voltage. From the experimental results, it is seen that the duty ratio of the clock cycle of SC circuit should be in the range from 0.05 to 0.70 . It is suggested that the proposed very low level dc current amplifier using SC circuit is an effective way to obtain both a faster output response and its miniaturization.

\section{Keywords}

dc Amplifier, Small Current Measurement, Switched Capacitor (SC) Circuit, SC Filter

\section{Introduction}

When very small currents are measured by mass spectroscopes and radiation detectors, response speeds of the measuring instruments are limited by those of very low level dc current amplifiers [1]. This means that the amplifiers are required to observe rapid transient phenomena. In general, the very low level dc current amplifier for measuring small currents consists of an amplifier having high input impedance and a high-ohmage negative feedback resistor. The amplifier with high-ohmage resistor has unavoidable effects of the stray capacitances across its terminals. This factor causes the amplifier to have a complicated frequency characteristic, which results in its poor responses [1] [2]. Some shielding techniques [3]-[5] have been reported for the purpose of de- 
creasing these capacitive components. In spite of the fact that these methods have been employed, it is difficult to realize drastic improvements of the response speeds of the very low level dc current amplifier. Neither are the amplifiers with shielding methods appropriate for miniaturization. A positive feedback circuit [6] had also been used as another approach to decreasing the stray capacitances. The amplifier with the positive feedback circuit however is unstable and begins to oscillate in this case. The resultant high speed response of the amplifier has not been achieved so far.

In this paper, an amplifier with switched capacitor (SC) circuit and offset controller are proposed. The SC circuit is equivalent to a resistor and is suitable for miniaturization. We investigated how much effect parasitic capacitances in the SC circuit have on the amplifier's output. Furthermore, effect of duty ratio of the clock cycle on the output of the amplifier was experimentally demonstrated.

\section{Circuit Analysis}

\subsection{Circuit Description}

Figure 1 depicts a very low level dc current amplifier, including SCF and a small current source. $C_{g}, R_{g}$ and $K$ are the input capacitance, input resistance, and amplification factor of the amplifier having a high input resistance, respectively. As an input signal to the amplifier in our experiment, we utilize a triangular wave voltage produced by the function generator $V_{g}$ and the differentiating capacitor $C_{s}$ (reactance attenuator) to obtain a square wave current $I_{s}$ with a high output impedance. $C_{o}$ is the output capacitance to the ground of $C_{s}$. The input stage of the offset controller is composed of a JFET which has much higher input impedance than the negative feedback circuit has. Its voltage drift is very small (several $\mu \mathrm{V}$ ). Therefore, the offset controller does not have much effect on the current detection sensitivity of the amplifier. The SC negative feedback circuit and SCF are shown in Figure 1(b). The former circuit is composed of a basic SC circuit and a feedback rate attenuator. The switches in Figure 1(b) are controlled by two non-overlapping clock signals. The switch $S_{11}$ is synchronous with $S_{12}$ and $S_{13} . S_{21}$ is synchronous with $S_{22}, S_{23}$ and $S_{24}$. The switches $S_{31}$ and $S_{32}$ in the SCF are synchronous with $S_{11}$ and $S_{21}$, respectively.

\subsection{Equivalent Resistance of SCNF}

From Figure 1(b), the voltage at node $b, V_{b}$, is given by

$$
V_{b}=\frac{C_{3}}{C_{2}+C_{3}} V_{o}
$$

and an electric charge $q_{1}$ at $C_{1}$ is

$$
q_{1}=C_{1}\left(V_{b}-V_{i}\right) .
$$

From Equation (1) and the relationship that $V_{o}=-K V_{i}$, the electric charge $q_{1}$ at $C_{1}$ can be rewritten as

$$
q_{1}=C_{1}\left(\frac{C_{3}}{C_{2}+C_{3}} V_{o}+\frac{V_{o}}{K}\right) \approx C_{1} V_{o} \frac{C_{2}+K C_{3}}{K\left(C_{2}+C_{3}\right)}
$$

for $K \gg 1$. The quantity of the charge that is transported from node $a$ to node $b$ is equivalent to $q_{1}$ because

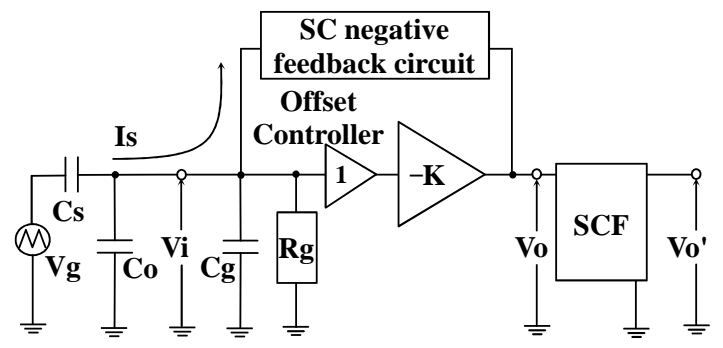

(a)

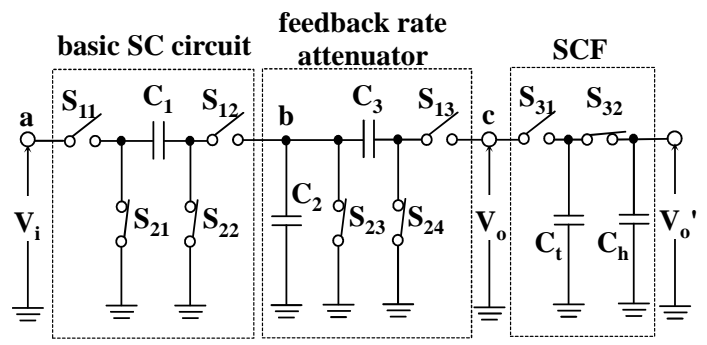

(b)

Figure 1. Circuit configurations of (a) very low level dc current amplifier; (b) SC negative feedback circuit and SCF. SC stands for switched capacitor. 
the electric charge $q_{1}$ at $C_{1}$ during $T_{2}$ is totally discharged. Thus, a current, $I$, flowing from node $a$ into node $b$ during one clock cycle $T_{s}$ is

$$
I=\frac{q_{1}}{T_{s}}=V_{o} \frac{C_{1}}{T_{s}} \cdot \frac{C_{2}+K C_{3}}{K\left(C_{2}+C_{3}\right)} .
$$

Since the current to be measured in the amplifier $I_{s}$ flows into the SC circuit, $I_{s}=I$. From the relationship that $V_{o}=R_{\text {feq }} I_{s}$, the equivalent resistance of SC negative feedback circuit $R_{\text {feq }}$ is represented by

$$
R_{\text {feq }}=\frac{T_{s}}{C_{1}} \cdot \frac{K\left(C_{2}+C_{3}\right)}{C_{2}+K C_{3}}
$$

while the equivalent SC resistance [7] $R_{s c}$ becomes

$$
R_{s c}=\frac{T_{s}}{C_{1}}=\frac{1}{C_{1} f_{s}}
$$

where $f_{s}$ is the clock frequency. The attenuation factor of the attenuator $x$ [8] is given by

$$
x=\frac{C_{2}+K C_{3}}{K\left(C_{2}+C_{3}\right)} \text {. }
$$

Thus, from Equations (4) to (6), $R_{\text {feq }}$ can be obtained as

$$
R_{\text {feq }}=\frac{1}{x C_{1} f_{s}}=\frac{R_{s c}}{x} .
$$

It is observed from Equation (6) that $x$ is dependent on the ratio of capacitances of $C_{2}$ and $C_{3}$.

\subsection{Theoretical Output Voltage of the Amplifier}

The equivalent SC negative feedback circuit is illustrated in Figure 2(a). It is seen from Equations (5) and (7) that the SC negative feedback circuit is equivalent to the capacitor of $x C_{1}$ and four switches $S_{11}, S_{13}, S_{21}$, and $S_{24}$. The equivalent circuit of the very low level dc current amplifier is shown in Figure 2(b). The box labeled "SC" in Figure 2(b) stands for the SC negative feedback circuit. The figure shows that the equivalent SC negative feedback circuit is connected with the equivalent circuit of the amplifier at the terminals between nodes $a$ and $c$.

Applying Millman's theorem to Figure 2(b), the input voltage $V_{i}$ is represented by

$$
V_{i}=\frac{I_{s}+j \omega x C_{1}\left(-K V_{i}\right)}{1 / R_{g}+j \omega\left(C_{i}+x C_{1}\right)}
$$

and

$$
C_{i}=C_{o}+C_{g}
$$

where $\omega$ is the angular frequency. The input admittance of the very low level dc current amplifier $Y_{\text {in }}$ is

$$
Y_{i n}=\frac{I_{s}}{V_{i}} \approx \frac{1}{R_{g}}+j \omega\left(C_{i}+x K C_{1}\right)
$$

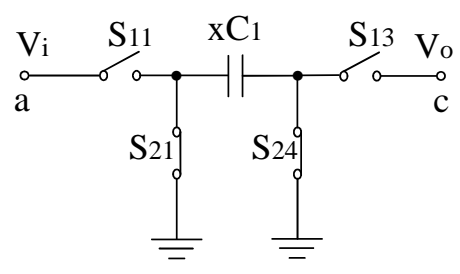

(a)

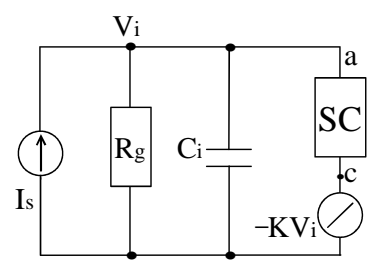

(b)

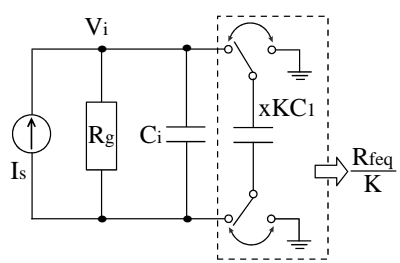

(c)

Figure 2. Equivalent circuits of (a) SC negative feedback circuit and (b) very low level dc current amplifier. (c) shows simplified input equivalent circuit of (b). 
for $K \gg 1$. Using Equation (8), a simplified input equivalent circuit of the very low level dc current amplifier using SC circuit can be drawn as shown in Figure 2(c).

An enlarged input voltage waveform of the amplifier at the positive final steady-state, $V_{i}$, is illustrated with the help of clock waveform in Figure 3. $T_{s}$ is the clock cycle of the switches. $T_{1}, T_{2}$ and $d$ are $(1-d) T_{s}$, $d T_{s}$, and a duty ratio of the clock cycle, respectively. Let the input voltage of the amplifier at $t=n T_{s}$ be $V_{i}(n)$. Subscript symbols “+” and "--” indicate just after and just before the time event occurs, respectively. For example, $V_{o}(n+1)_{-}$means the voltage just before $t=(n+1) T_{s}$. The amplitude of the input voltage for a cycle, $V_{m}$, is

$$
V_{m}=\frac{1}{C_{i}+x K C_{1}} \int_{n T_{s}}^{\left(n+(1-d) T_{s}\right.} I_{s} \mathrm{~d} t+\frac{1}{C_{i}} \int_{(n+(1-d)) T_{s}}^{(n+1) T_{s}} I_{s} \mathrm{~d} t=\frac{C_{i}+d x K C_{1}}{C_{i}\left(C_{i}+x K C_{1}\right)} I_{s} T_{s} .
$$

Since electric charges of the SC circuit are conserved just before and after $t=n T_{s}$, the following equation is obtained

$$
C_{i} V_{i}(n)_{-}=\left(C_{i}+x K C_{1}\right) V_{i}(n)_{+} .
$$

The input voltage just before $t=n T_{s}$ is

$$
V_{i}(n)_{-}=\left(1+\frac{x K C_{1}}{C_{i}}\right) V_{i}(n)_{+} .
$$

From Figure 3 and Equation (10), the voltage $V_{m}$ is

$$
V_{m}=V_{i}(n)_{-}-V_{i}(n)_{+}=\frac{x K C_{1}}{C_{i}} V_{i}(n)_{+} .
$$

From Equations (9) and (11), the input voltage just after $t=n T_{s}$ is given by

$$
V_{i}(n)_{+}=\frac{I_{s} T_{s}}{x K C_{1}} \cdot \frac{C_{i}+d x K C_{1}}{C_{i}+x K C_{1}} .
$$

The resultant peak voltage $V_{i p 1}$ during $T_{1}$ is

$$
V_{i p 1}=V_{i}(n)_{+}+\frac{1}{C_{i}+x K C_{1}} \int_{n T_{s}}^{(n+(1-d)) T_{s}} I_{s} \mathrm{~d} t=V_{i}(n)_{+}+\frac{1-d}{C_{i}+x K C_{1}} I_{s} T_{s} .
$$

Substituting Equation (12) into Equation (13) gives the following equation:

$$
V_{i p 1}=\frac{I_{s} T_{s}}{x K C_{1}}=\frac{R_{\text {feq }}}{K} I_{s} .
$$

Therefore, the peak output voltage of the amplifier during $T_{1}, V_{o p 1}$, can be written as

$$
V_{o p 1}=-K V_{i p 1}=-R_{f e q} I_{s} \text {. }
$$

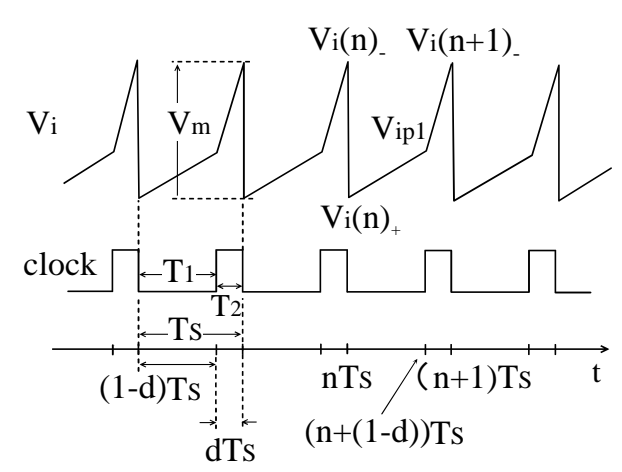

Figure 3. Relationship between enlarged input voltage and clock waveform. 
It is found from Equation (15) that the theoretical output voltage of the very low level dc current amplifier using SC circuit can be obtained by sampling $V_{o p 1}$. In this paper, the SCF is used to sample $V_{o p 1}$ from the output voltage of the very low level dc current amplifier using SC circuit for the following reasons. Using a sampleand-hold circuit generally requires a clock generator that completely differs from two non-overlapping clock signals utilized by the SC negative feedback circuit. Using a low-pass filter provides for not theoretical output voltage, but approximately half amplitude of output voltage of the amplifier at a final steady-state. On the other hand, using the SCF with the SC circuit allows for sharing the two non-overlapping clock signals. In addition, both the SCF and SC circuit can be manufacturable by the same process. We are easily available to miniaturize SC circuits using IC-compatible techniques. Therefore, the SCF is useful from the viewpoint of miniaturization.

\section{Methods}

\subsection{Effect of Parasitic Capacitances on the Amplifier's Output}

To evaluate response speed of the very low level dc current amplifier, a square wave current $I_{s}$ with a time period of $5 \mathrm{~ms}$ and an amplitude of $10 \mathrm{nA}$ was input to the amplifier. $K$ and $C_{g}$ were set to 1300 and $17 \mathrm{pF}$, respectively. We fixed the equivalent SC resistance $R_{R C}$ of $1 \mathrm{M} \Omega$ using $C_{1}$ of $10 \mathrm{pF}$ and $f_{s}$ of $100 \mathrm{kHz}$. The attenuation factor $x$ of $1 / 100$ was also set using both $C_{2}$ of $1000 \mathrm{pF}$ and $C_{3}$ of $9.3 \mathrm{pF}$. The total equivalent resistance $R_{\text {feq }}$ of the SC negative feedback circuit was $100 \mathrm{M} \Omega$. The duty ratio $d$ of 0.5 was used. A switch model [9] used in the computer simulation is shown in Figure 4. The symbols $D, G$, and $S$ stand for drain, gate, and source of MOS-FETs. Assuming that parasitic capacitances between two terminals exist, as shown in Figure 4(a) and Figure 4(b), each analog switch composed of a combination of an nMOS and pMOS was used (see Figure 4(c)). Transient analyses of the very low level dc current amplifier using SC circuit were carried out using the electronic circuit simulator PSpice (Cadence Design System, Inc.). Table 1 lists the parasitic capacitance values determined by trial and error.

\subsection{Effect of Duty Ratio on the Amplifier's Output}

The amplification factor $K$ of the very low level dc current amplifier using SC circuit shown in Figure 1 was set to $62.3 \mathrm{~dB}$. Its output waveform was observed using an oscilloscope. Since the triangular wave voltage, which had a time period of $10 \mathrm{~ms}$ and an amplitude of $10 \mathrm{~V}$, was differentiated by the differentiating capacitor $C_{s}$ of $1.25 \mathrm{pF}$, a square wave current with a time period of $10 \mathrm{~ms}$ and an amplitude of $10 \mathrm{nA}$ was obtained as an input current $I_{s}$ to the amplifier. As switches for the SC circuit and SCF, we used CMOS analog switches

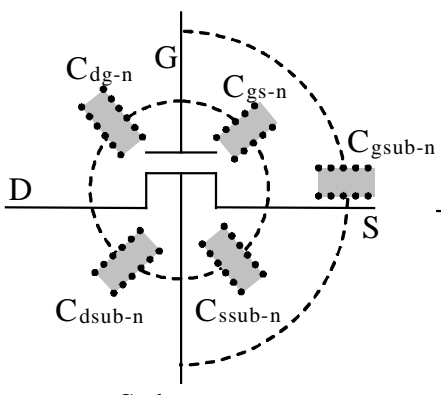

Substrate

(a)

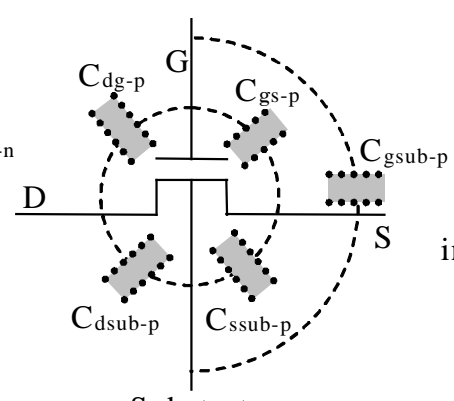

Substrate

(b)

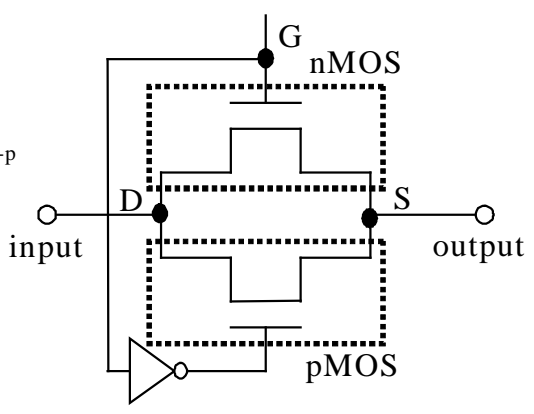

(c)

Figure 4. Switch model used in PSpice simulation. Configurations of (a) nMOS, (b) pMOS FET models with parasitic capacitances, and (c) CMOS switch.

Table 1. Parasitic capacitance values based on the assumption that nMOS and pMOS have the same parasitic capacitive components.

\begin{tabular}{ccccc}
\hline \multicolumn{5}{c}{ Parasitic capacitance [pF] } \\
\hline$C_{d g-n}, C_{d g-p}$ & $C_{g s-n}, C_{g s-p}$ & $C_{d s u b-n}, C_{d s u b-p}$ & $C_{\text {ssub-n }}, C_{\text {ssub-p }}$ & $C_{g s u b-n}, C_{g s u b-p}$ \\
1.0 & 0.9 & 0.7 & 0.7 & 0.6 \\
\hline
\end{tabular}


(MAX326, MAXIM Integrated Products, Inc.) having the maximum leakage current of $10 \mathrm{pA}$. Further, variable capacitors $C_{1}$ and $C_{3}$ were utilized. Parasitic capacitances of analog switches have some effect on equivalent resistance of the SC negative feedback circuit $R_{\text {feq }}$, which causes errors in $R_{\text {feq }}$ of the amplifier. Thus, the equivalent SC resistance $R_{s c}$ with the clock frequency $f_{s}$ of $100 \mathrm{kHz}$ was set to $1 \mathrm{M} \Omega$ by adjusting capacitance of $C_{1}$, and then the attenuation factor of the attenuator $x$ was set to $1 / 100$ by adjusting capacitance of $C_{3}$. Referring to Equation (7), the total equivalent resistance of the SC circuit became $100 \mathrm{M} \Omega$. An offset voltage controller, which was connected to the input of the amplifier and had a gain of unity, was also used to cancel the offset voltage in our experiment.

\section{Results and Discussions}

\subsection{Effect of Parasitic Capacitances on the Amplifier's Output}

First, based on the assumption that nMOS has exactly the same parasitic capacitances as pMOS has, transient analyses of the amplifier were done. Figure 5 shows the simulation result with the parasitic capacitances shown in Table 1. It can be observed that the output waveforms of the amplifier have vibrations that cause black area due to charge and discharge actions of the SC negative feedback circuit (see Figure 5(a) and Figure 5(b)). Thus, it is difficult to measure an input current from them. Calculating average values of $V_{o p 1}$ from 10.0 ms to 12.5 $\mathrm{ms}$ and from $12.5 \mathrm{~ms}$ to $15.0 \mathrm{~ms}$ in Figure 5(a), they are respective $+1.0 \mathrm{~V}$ and $-1.0 \mathrm{~V}$. From Equation (15), output voltage of $1 \mathrm{~V}$ should be obtained as the theoretical output of the amplifier. The output waveform of the SCF, $V_{o}^{\prime}$, is shown in Figure 5(c). In this case, the peaks of the output voltage during $T_{1}$ were sampled by the SCF. As generally defined, the rise time is the time required for the output waveform to rise from $10 \%$ to $90 \%$ of its final steady-state value. The rise time of the output waveform of the SCF is $10.3 \mu \mathrm{s}$, while that of the amplifier using conventionally used high-ohmage resistor is $83.8 \mu$ s [10]. It is seen from Figure 5(c) that using the

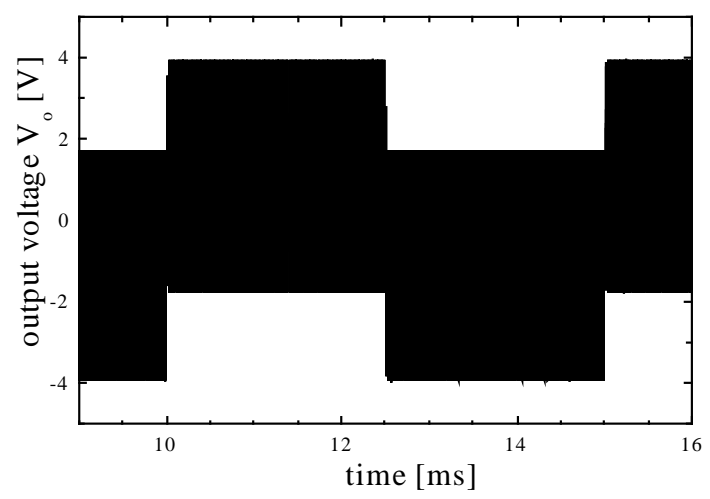

(a)

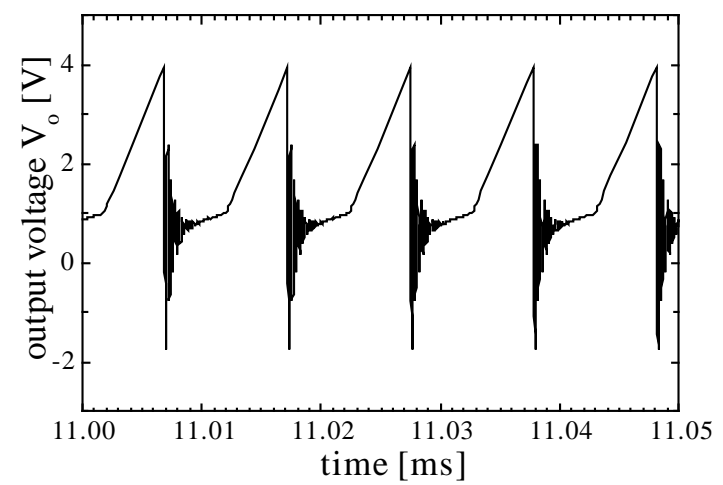

(b)

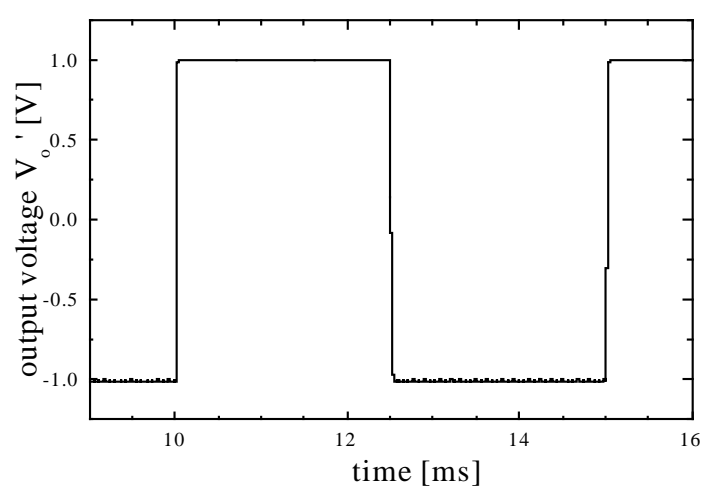

(c)

Figure 5. Simulation results with parasitic capacitances shown in Table 1. (a) Output waveform of very low level dc current amplifier using SC circuit; (b) its enlarged waveform at a positive final steady-state; and (c) output waveform of SCF. The rise time in (c) is $10.3 \mu \mathrm{s}$. 
SCF considerably reduces vibrations as well as unnecessary components, and that the input current $I_{s}$ can be obtained by measuring the amplitude of its output voltage.

Secondly, we also performed computer simulations with an addition of $0.5 \mathrm{pF}$ to each parasitic capacitance of nMOS or pMOS listed in Table 1 to find out which parasitic capacitance would have effect on the output of the amplifier. Table 2 summarizes parasitic capacitances that have effect on offset voltage of the amplifier. For example, all the cases in the switch $S_{11}$ that $C_{d g-n}(1.5 \mathrm{pF})>C_{d g-p}(1.0 \mathrm{pF}), C_{d g-p}(1.5 \mathrm{pF})>C_{d g-n}(1.0 \mathrm{pF})$, $C_{g s-n}(1.4 \mathrm{pF})>C_{g s-p}(0.9 \mathrm{pF})$, and $C_{g s-p}(1.4 \mathrm{pF})>C_{g s-n}(0.9 \mathrm{pF})$ cause generation of offset voltages at the amplifier's output. On the other hand, parasitic capacitances that are not listed in Table 2 do not have effect on its output voltage. From the simulation results, it is found that differences between value of $C_{d q-n}$ and that of $C_{d g-p}$ in $S_{11}, S_{12}, S_{21}, S_{22}$, and $S_{23}$, and between that of $C_{g s-n}$ and that of $C_{g s-p}$ in $S_{11}$ result in generating offset voltages of the amplifier, and that parasitic capacitive components that are distributed close to the amplifier's input portion are deeply related to it.

\subsection{Effect of Duty Ratio on the Amplifier's Output}

Experimental results are shown in Figure 6. In the experimental result with the duty ratio of $d=0.05$, the output amplitude of the amplifier is larger than $1 \mathrm{~V}$. It is also observed that the output waveform at positive and negative final steady-states is rather distorted. On the other hand, in that of $d=0.70$, the amplitude becomes smaller than $1 \mathrm{~V}$. It is found from the experimental results that the duty ratio of the clock cycle should be in the range: $0.05<d<0.70$. Using duty ratios larger than 0.70 leads to output waveform degradation. It is thought that the longer $T_{1}$ (the shorter $d$ ) we use, the more stable output waveform can be sampled using the SCF.

Finally, a relationship between the clock frequency $f_{s}$ and error rate of $R_{\text {feq }}$ was investigated. Setting $f_{s}$ and $C_{2}$ to respective $100 \mathrm{kHz}$ and $1000 \mathrm{pF}$, we adjusted both capacitances of $C_{1}$ and $C_{3}$ to precisely obtain $R_{\text {feq }}$ of $100 \mathrm{M} \Omega$, and then changed $f_{s}$ ranging from $50 \mathrm{kHz}$ to $200 \mathrm{kHz}$ when $I_{s}=5 \mathrm{nA}, I_{s}=10 \mathrm{nA}$, and $I_{s}=20 \mathrm{nA}$, respectively. The equivalent resistance of $R_{f e q}$ was obtained by measuring output voltage of the SCF. The relationship between $f_{s}$ and error rate of $R_{\text {feq }}$, with $x$ of $1 / 100$, is shown in Figure 7. Referring to Equation (7), $R_{\text {fea }}$ is inversely proportional to $f_{s}$. This means that decreasing $f_{s}$ is equivalent to increasing $R_{\text {feq }}$. It is seen from Figure 7 that there is a tendency for the error rate to decrease as $R_{\text {feq }}$ increases. It is thought that the stable output waveform will get longer as $T_{s}$ increases because of charge action of the SC circuit.

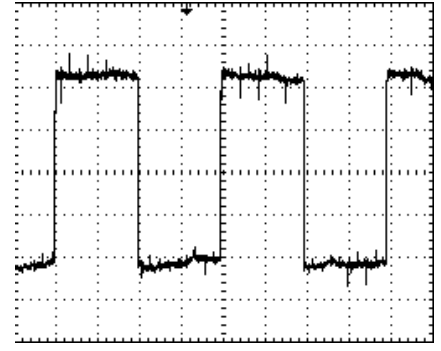

(a)

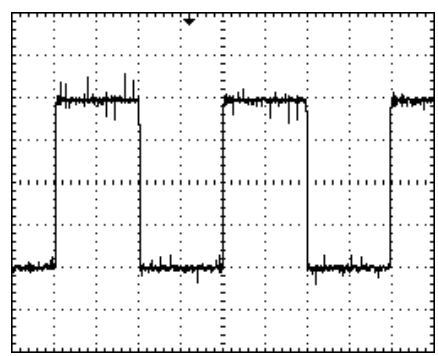

(c)

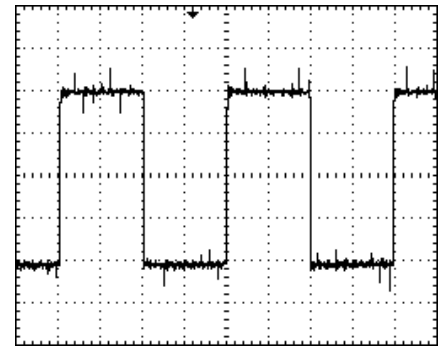

(b)

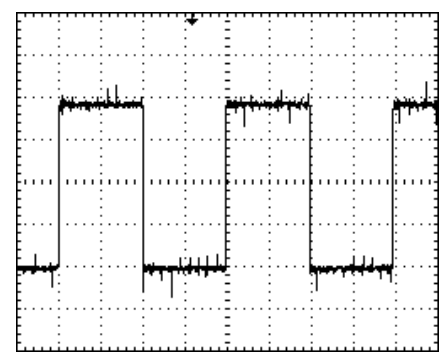

(d)

Figure 6. Output waveforms of the SCF with duty ratios of (a) $d=0.05$; (b) $d=0.10$; (c) $d=0.50$; and (d) $d=0.70$, respectively. Scale: H: 2.5 $\mathrm{ms} / \mathrm{div}, \mathrm{V}: 0.5 \mathrm{~V} / \mathrm{div}$. 


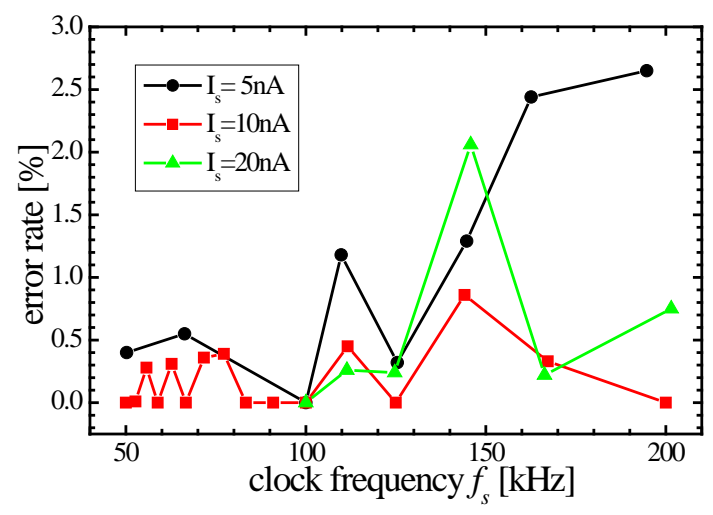

Figure 7. Relationship between the clock frequency $f_{s}$ and error rate of $R_{\text {feq }} \quad(x=1 / 100)$.

Table 2. Parasitic capacitances in each switch that have effect on offset voltage of the amplifier.

\begin{tabular}{|c|c|c|c|c|}
\hline \multicolumn{5}{|c|}{ Parasitic capacitance $[\mathrm{pF}]$} \\
\hline$S_{11}$ & $S_{12}$ & $S_{21}$ & $S_{22}$ & $S_{23}$ \\
\hline$C_{d g-n}, C_{d g-p}, \quad C_{g s-n}, C_{g s-p}$ & $C_{d g-n}, C_{d g-p}$ & $C_{d g-n}, C_{d g-p}$ & $C_{d q-n}, C_{d q-p}$ & $C_{d g-n}, C_{d g-p}$ \\
\hline
\end{tabular}

\section{Conclusion}

It is found from the simulation results that the parasitic capacitive components that are distributed close to the input portion of the amplifier have effect on the offset voltage. The experimental results show that the duty ratio of the clock cycle has an effective range. The error rate of less than $3.0 \%$ in $R_{f e q}$ is also obtained in our experiment. These results suggested that the proposed amplifier using SC circuit would provide the measuring device having better properties of both faster response and downsizing.

\section{Acknowledgements}

We would like to thank anonymous referees for their valuable comments and suggestions.

\section{References}

[1] Goto, K. and Ishikawa, K. (1979) Design and Construction of High Speed Pico-Ammeter. The Journal of the Vacuum Society of Japan, 22, 235-246. (in Japanese) http://dx.doi.org/10.3131/jvsj.22.235

[2] Nakamura, I. and Kano, T. (1983) Noise and Fast Response of a Very Small dc Current Amplifier. Oyo Buturi, 52, 330-338. (in Japanese)

[3] Galliana, F. and Capra, P.P. (2010) Hamon $10 \times 100 \mathrm{M} \Omega$ Resistor Based Traceable Source for Calibration of Picoammeters in the Range 100 pA - $100 \mathrm{nA}$. Measurement, 43, 1277-1281. http://dx.doi.org/10.1016/j.measurement.2010.07.006

[4] Galliana, F., Capra, P.P. and Gasparotto, E. (2009) Metrological Management of the High dc Resistance Scale at INRIM. Measurement, 42, 314-321. http://dx.doi.org/10.1016/j.measurement.2008.07.002

[5] Nakamura, I. and Takemura, M. (1989) Fast Response of a Very Low-Level dc Current Amplifier Using Improvement of Feedback Resistor Shielding Structure. IEICE Transactions on Electronics, J72-C-II, 885-892. (in Japanese)

[6] Nakamura, I. and Kano, T. (1985) High Speed Response of a Very Low Level dc Current Amplifier Using Positive Feedback Loop. Oyo Buturi, 54, 945-951. (in Japanese)

[7] Grebene, A.B. (1984) Bipolar and MOS Analog Integrated Circuit Design. John Wiley \& Sons Inc., Hoboken.

[8] Higa, H., Onaga, R., Nakamura, N. and Nakamura, I. (2005) A Basic Study on a Very Low-Level Dc Current Amplifier Using a Switched-Capacitor Circuit: Comparison between Simulation and Experimental Results. Proceeding of the ITC-CSCC2005, Jeju, 3-7 July 2005, 1133-1134. 
[9] Weste, N.H.E. and Eshraghian, K. (1999) Principle of CMOS VLSI Design: A Systems Perspective, Maruzen (tr. Tomisawa, T. and Matsuyama, Y.).

[10] Higa, H., Nakamura, N. and Nakamura, I. (2005) A Basic Study on a Very Low-Level dc Current Amplifier Using a Switched-Capacitor Circuit. IEICE Transactions on Fundamentals, E88-A, 1394-1400. 
Scientific Research Publishing (SCIRP) is one of the largest Open Access journal publishers. It is currently publishing more than 200 open access, online, peer-reviewed journals covering a wide range of academic disciplines. SCIRP serves the worldwide academic communities and contributes to the progress and application of science with its publication.

Other selected journals from SCIRP are listed as below. Submit your manuscript to us via either submit@scirp.org or Online Submission Portal.
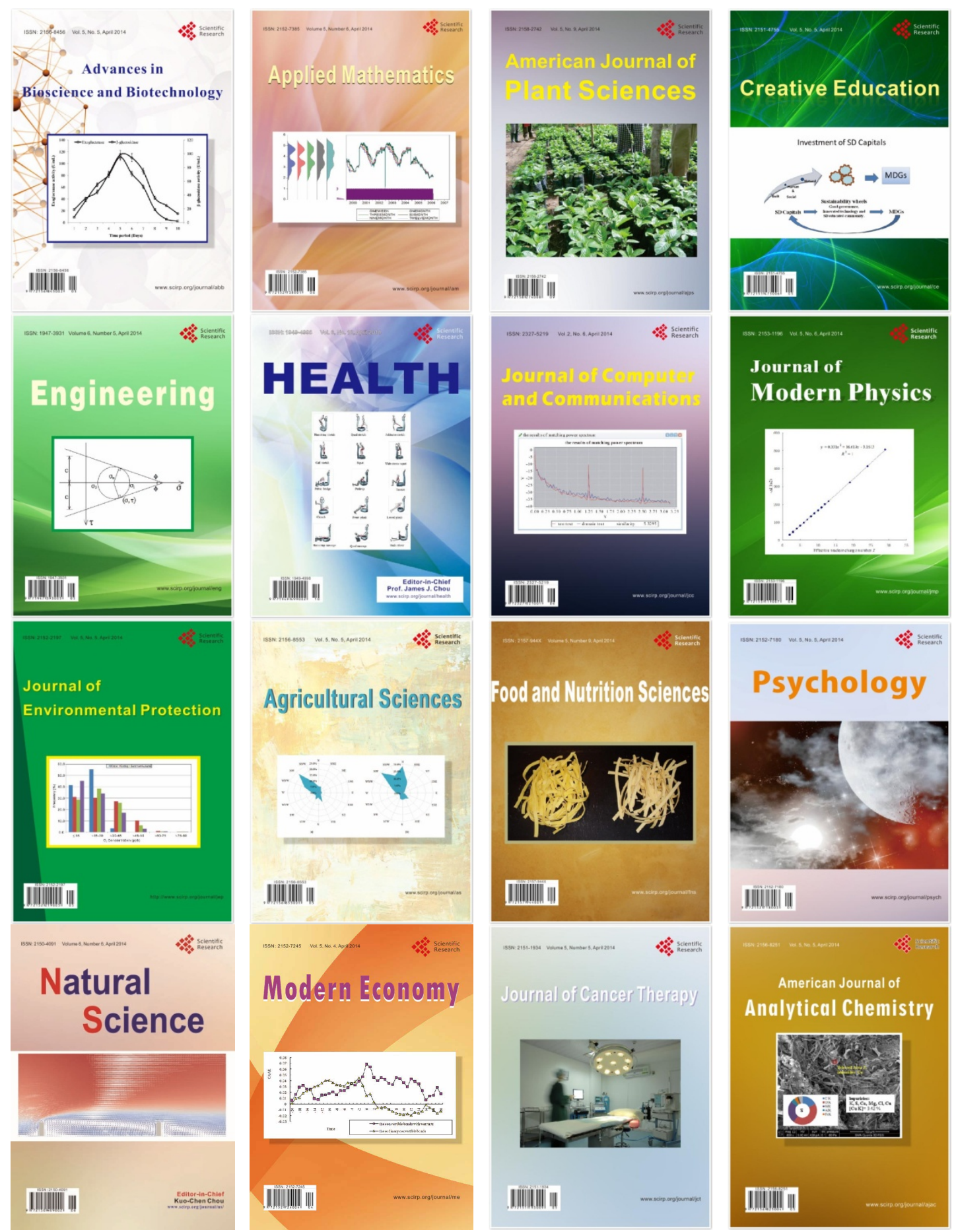